\title{
Numerical Simulation of Viscous Fingering in Non-Newtonian Fluids in a Hele-Shaw Cell
}

\author{
Takehiro Yamamoto, Hiroo Kamikawa, Noriyasu Mori, and Kiyoji NaKamura \\ Department of Mechanophysics Engineering, Graduate School of Engineering, \\ Osaka University, 2-1, Yamadaoka, Suita, Osaka 565-0871, Japan \\ (Received : October 31, 2001)
}

\begin{abstract}
Viscous fingering in non-Newtonian fluids in a rectangular Hele-Shaw cell was numerically simulated using the finite element method together with the volume of fluid (VOF) method for the representation of an interface between two viscous fluids. Interfacial tension was treated as a volumeric force using the continuum surface force (CSF) model. The Carreau model was applied for describing the shear-thinning viscosity. In addition, the viscous fingering in a Newtonian fluid was also calculated for comparison. The computation was performed under various conditions of initial finger shape, interfacial tension, and mean velocity. The typical patterns such as 'shielding', 'spreading', and 'splitting' were simulated and the numerical results predicted that the shear-thinning viscosity increased the shielding effect. The present results indicated the numerical approach was effective.
\end{abstract}

Key Words: Viscous fingering / Non-Newtonian fluid / Free surface flow / Hele-Shaw cell / Numerical simulation

\section{INTRODUCTION}

When a less viscous fluid replaces a more viscous fluid in a thin layer between a pair of parallel plates, the interface between two fluids forms a complex pattern. This phenomenon is called viscous fingering because the pattern looks similar to fingers. The fingers unstably grow repeating three typical patterns such as spreading, splitting, and shielding. The viscous fingering has been studied in various fields by both experimental and numerical approach, e.g., Refs.1)-4) and the references therein.

The numerical approach is effective for the analysis of mechanism of change in growth patterns. In the previous numerical studies, the problem was formulated in a simple form under some approximations and the stability of the interface was mainly discussed. However, some numerical simulations were attempted using $\mathrm{BEM}^{5)}$ and $\mathrm{FEM}^{6)}$ to predict the process of the finger growth.

In plastic industry, non-Newtonian viscous fingers are observed in the polymer processing such as the gas-assisted injection molding and affect the quality of products. ${ }^{7)}$ In recent studies of viscous fingering in non-Newtonian fluids, many researchers indicated the effect of shear-thinning viscosity and/ or elastic properties on the growth of viscous fingers, e.g. Refs. 8)-20). Hence it is important to study the relationship between the finger growth and the non-Newtonian property of polymeric liquids and to clarify the mechanism of finger growth.

In theoretical study of viscous fingering in non-Newtonian fluids, the surface stability problem and the growth of single finger are mainly analyzed., ${ }^{3,18,19)}$ In the analysis, theoretical prediction of the finger width and the tip-velocity for Newtonian fluids is modified by replacing the Newtonian viscosity by the shear rate dependent viscosity. The modified theory for shear-thinning fluids predicts that the finger width is thinner than that in Newtonian fluids and the tip-velocity nonlinearly depends on the pressure gradient. These predictions qualitatively agree with experimental observations. Lindner et al. ${ }^{19)}$ recently proposed some modifications for strongly shearthinning fluids and provided better description of the finger behavior.

To the authors' knowledge, few theoretical or numerical studies of viscous fingering in non-Newtonian fluids treated branching fingers though they often appear in experiments and are interesting phenomena. In the present study, we numerically simulated the viscous fingering in non-Newtonian fluids in a Hele-Shaw cell using the finite element method to investigate the mechanism of the finger growth accompanying typical growth patterns such as spreading, splitting, and shielding. The Carreau model was applied for modeling the shear-thinning viscosity of non-Newtonian fluids. 


\section{BASIC EQUATION}

In the present simulation, the flow between plates separated by a narrow gap was considered, thus the flow can be approximated as a Hele-Shaw flow. Figure 1 illustrates a schematic diagram of viscous fingering in a Hele-Shaw.

The equation of continuity is expressed in terms of the average velocity, $\bar{u}$ and $\bar{v}$ as follows:

$$
\begin{aligned}
& \frac{\partial(h \bar{u})}{\partial x}+\frac{\partial(h \bar{v})}{\partial y}=0 \\
& \bar{u}(x, y)=\frac{1}{h} \int_{0}^{h} u(x, y, z) d z \\
& \bar{v}(x, y)=\frac{1}{h} \int_{0}^{h} v(x, y, z) d z
\end{aligned}
$$

where $h$ is the half thickness of the cell, and $u$ and $v$ are the velocities in the $x$ - and $y$-direction, respectively. The velocity in the $z$-direction is 0 under the Hele-Shaw approximation. The equation of motion is expressed as follows:

$$
\begin{aligned}
& \frac{\partial}{\partial z}\left(\eta \frac{\partial u}{\partial z}\right)=\frac{\partial P}{\partial x}-F_{x} \equiv \Lambda_{x} \\
& \frac{\partial}{\partial z}\left(\eta \frac{\partial v}{\partial z}\right)=\frac{\partial P}{\partial y}-F_{y} \equiv \Lambda_{y}
\end{aligned}
$$

where $F_{x}$ and $F_{y}$ are the $x$ and $y$ components of the body force, respectively. $P$ denotes the pressure. In the Hele-Shaw approximation, the pressure is uniform in the $z$-direction.

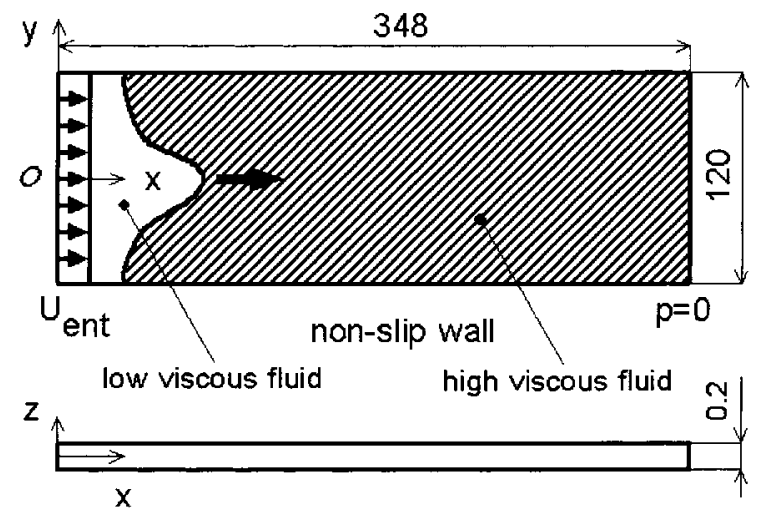

Fig.1 Schematic diagram of rectangular Hele-Shaw cell and coordinate system.
Integrating Eqs. (4) and (5) with respect to $z$ under the boundary conditions, $\partial u / \partial z=\partial v / \partial z=0$ at $z=0$ and $u=v=0$ at $z= \pm h$, yields the following expression of $u, v, \bar{u}$, and $\bar{v}$ :

$$
u(x, y, z)=-\Lambda_{x} \int_{z}^{h} \frac{\zeta}{\eta} d \zeta
$$

$$
v(x, y, z)=-\Lambda_{y} \int_{z}^{h} \frac{\zeta}{\eta} d \zeta
$$

$$
\bar{u}(x, y)=\frac{S}{h} \Lambda_{x}
$$

$$
\bar{v}(x, y)=\frac{S}{h} \Lambda_{y}
$$

where $S$ is fluidity defined by

$$
S=\int_{0}^{h} \frac{z^{2}}{\eta} d z
$$

Substituting Eqs. (8) and (9) into Eq. (1), one obtains the following equation:

$$
\frac{\partial}{\partial x}\left(-S \Lambda_{x}\right)+\frac{\partial}{\partial y}\left(-S \Lambda_{y}\right)=0
$$

In the present study, the fluid that fills the cell is nonNewtonian. We applied the Carreau model (12) to describe the shear-thinning viscosity of the more viscous fluid.

$$
\frac{\eta-\eta_{\infty}}{\eta_{0}-\eta_{\infty}}=\left\{1+(\lambda \dot{\gamma})^{2}\right\}^{\frac{n-1}{2}}
$$

where $\eta_{0}$ is the zero-shear-rate viscosity, $\eta_{\infty}$ the infinite-shearrate viscosity, $\lambda$ the relaxation time, $n$ the power index. The shear rate $\dot{\gamma}$ is defined by

$$
\dot{\gamma}=\sqrt{2 \operatorname{tr}(D \cdot D)}
$$

where $\boldsymbol{D}=\left(\nabla \boldsymbol{v}+\nabla \boldsymbol{v}^{\mathrm{T}}\right) / 2$ is the rate-of-deformation tensor and $\boldsymbol{v}$ the velocity vector. 


\section{NUMERICAL SCHEME}

We numerically solved Eq. (11) to obtain the pressure field using the Galerkin finite element method. The computational domain is divided into a large number of triangular elements with 3 nodes and the pressure is interpolated by a linear function in the element.

The movement of the interface of the two fluids is calculated by VOF (volume-of-fluid) method. The control volume surrounding a node is defined as shown in Fig.2. The condition of the control volume is described by a function $\phi: \phi$ is the fractional volume of the high viscous fluid in a control volume and has a value between zero and unity. $\phi=0,1$ means the volume is fully filled with a low viscous fluid or a high viscous fluid, respectively. When $0<\phi<1$, the control volume belongs to the interface region. Following usual VOF-based computation, the contour line of $\phi=0.5$ is regarded as the interface in the present computation.

The movement of the interface is expressed by the following advection equation:

$$
\frac{\partial \phi}{\partial t}+\bar{u} \frac{\partial \phi}{\partial x}+\bar{v} \frac{\partial \phi}{\partial y}=0
$$

Under the assumption that $\bar{u}$ and $\bar{v}$ are constant during the fluid moves from $(x-\Delta x, y-\Delta y)$ to $(x, y)$ in a small time interval $\Delta t, \Delta x$ and $\Delta y$ are expressed as $\Delta x=\bar{u} \Delta t$ and $\Delta y=\bar{v} \Delta \mathrm{t}$. Consequently, Eq. (14) is reduced to

$$
\phi(x, y, t+\triangle t)=\phi(x-\bar{u} \triangle t, y-\bar{v} \triangle t, t)
$$

The transport of $\phi$ is calculated according to Eq. (15). The nodal velocities $\bar{u}$ and $\bar{v}$ are evaluated by the mean value of the average velocities in all the element which include the node.

The fluidity $S$ for each element was evaluated based on the mean value of $\phi$ on the three vertexes. The method for calculating $S$ differed from the region where the node belonged, i.e. the low viscous fluid, the high viscous fluid, or the interface.

The low viscous fluid is Newtonian, thus the viscosity $\eta$ is constant and $S$ is also constant, $S=h^{3} /(3 \eta)$. On the other hand, in the case of the high viscous fluid whose viscosity is described by the Carreau model, the viscosity depends on the shear rate. Consequently, shear rate is calculated from the velocity profile and the viscosity $\eta$ is evaluated. Then, the

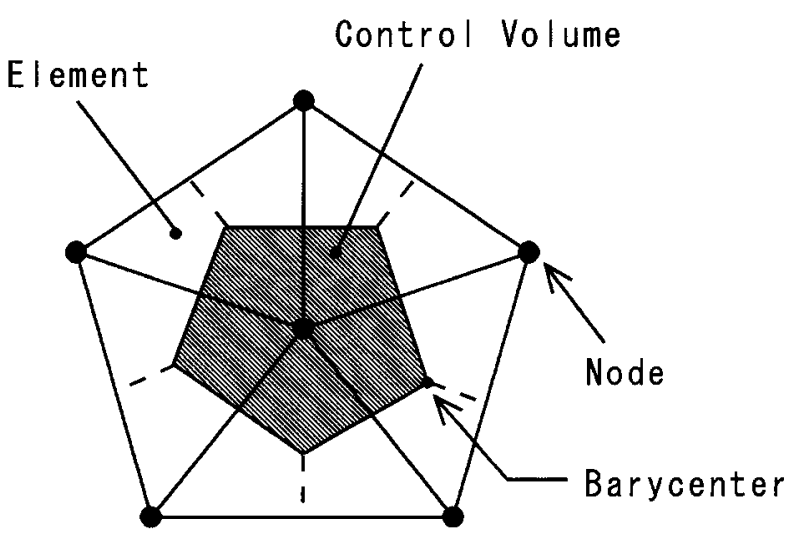

Fig.2 Control volume.

integral in Eq. (10) is carried out using $\eta$ obtained.

In the interface region, both the low and high viscous fluids exist. In this region, the value of $S$ is interpolated using the fluidity in the low viscous fluid region $S_{\ell}$ and that in the high viscous fluid region $S_{h}$ according to the following equations:

$$
S=\left\{\begin{array}{cc}
\phi S_{h}+(1-\phi) S_{\ell}, & (0 \leq \phi<0.5) \\
S_{h} . & (0.5 \leq \phi \leq 1)
\end{array}\right.
$$

If the linear interpolation (the first equation of (16)) is applied for all $\phi$, the value of $S$ gradually changes across the interface, especially when $S_{\ell}$ and $S_{h}$ are much different. The gradual change causes numerical diffusion of the interface ${ }^{12)}$, thus we introduced the interpolation of Eq. (16) in the present computation.

For introducing the effect of interfacial tension, the Continuum Surface Force (CSF) model ${ }^{21}$ was applied. Nakayama et al. ${ }^{6}$ indicated that FEM approach with the CSF model was capable for describing the growth of viscous fingers in Newtonian fluids.

In the CSF model, the interfacial force is translated into the body force $\boldsymbol{F}_{i v}$ as ${ }^{22)}$

$$
\boldsymbol{F}_{i v}=-\sigma \kappa \frac{\nabla \phi}{[\phi]}
$$

where $\sigma$ is the surface tension, $[\phi]$ denotes the amount of jump in $\phi$ across the interface and is equal to 1 in the present simulation. $\kappa$ is the curvature of the interface expressed as

$$
\kappa=-\nabla \cdot \hat{\boldsymbol{n}}
$$

where $\hat{\boldsymbol{n}}$ is a unit vector normal to the interface and is defined by $\hat{\boldsymbol{n}}=\boldsymbol{n} /|\boldsymbol{n}|$ and $\boldsymbol{n}=-\nabla \phi$. 
The following shows the procedure of the present computation:

(a) Set the initial finger shape.

(b) Calculate the interfacial force $\boldsymbol{F}_{i v}$ based on the CSF model, Eq. (17), and take it into the body force.

(c) Calculate the viscosity and velocity distributions in the $z$-direction for the Carreau model (in the high viscous fluid region).

(d) Calculate the fluidity $S$ in both the low and high viscous fluid regions. Interpolate the fluidity according to Eq. (16) for the element that belongs to the interface region.

(e) Calculate the pressure field by solving the finite element equation.

(f) Repeat from (b) to (e) until the solution converges.

(g) Calculate the mean velocities $\bar{u}$ and $\bar{v}$ in each element according to Eqs. (8) and (9).

(h) Move the interface using $\bar{u}$ and $\bar{v}$ obtained in (g).

(i) Repeat from (b) to (h) until the front of the finger reaches the end of the cell.

\section{NUMERICAL CONDITION}

\subsection{Test fluid}

The model parameters for the high viscous fluid used in the calculation are as follows: $\eta_{0}=130 \mathrm{~Pa} \bullet \mathrm{s}, \eta_{\infty}=0, \lambda=5 \mathrm{~s}, n=0.35$ for a non-Newtonian fluid and $\eta_{0}=130 \mathrm{~Pa} \bullet \mathrm{s}, \lambda=0, n=1$ for a Newtonian fluid. Figure 3 shows the steady shear viscosity of the test fluids. In the figure, viscosity of the $2.0 \mathrm{wt} \%$ aqueous solution of carboxymethylcellulose (CMC; Daicel Chemical

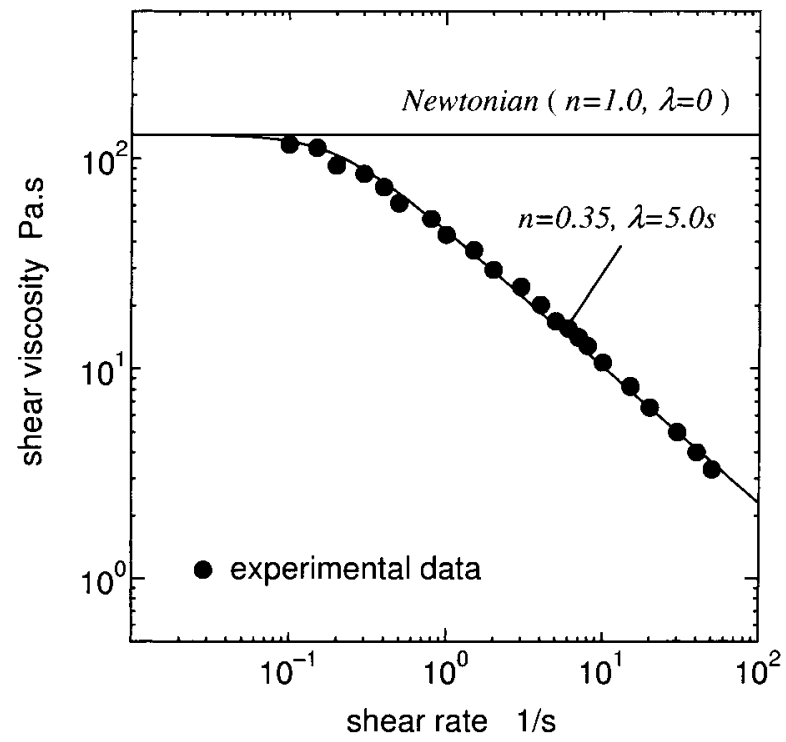

Fig.3 Steady shear viscosity of test fluids. • : experimental data of 2.0 $\mathrm{wt} \%$ aqueous solution of CMC.
Industries CMC DAICEL 2260) is shown; the data were measured with a cone-plate type rheometer (Nippon Rheology Equipment NRM-2000S-NF). As a low viscous fluid, we applied the viscosity of air: $\eta=1.81 \times 10^{-5} \mathrm{~Pa} \bullet$ s. The surface tension coefficient $\sigma$ of the $2.0 \mathrm{wt} \%$ aqueous solution of CMC measured with a Du Nouy type tensionmeter is $75 \mathrm{mN} / \mathrm{m}$.

\subsection{Cell geometry and boundary condition}

The numerical simulation was carried out for a rectangular cell of $120 \mathrm{~mm}$ in width, $348 \mathrm{~mm}$ in length, and $0.2 \mathrm{~mm}$ in thickness (Fig.1). The geometry is the same as that used in our previous experimental study. ${ }^{15)}$ Figure 4 shows the finite element mesh for the computation. The mesh is consist of 4797 nodes and 9280 elements. As boundary conditions, the mean velocity $U_{\text {ent }}$ is given at the cell entrance, $P=0$ at the exit, and no-slip condition is applied on the cell wall.

\section{RESULTS AND DISCUSSION}

\subsection{Finger growth}

The initial interface profiles considered are shown in Fig.5. The profiles of the single-protuberance and the doubleprotuberances are labeled ' $\mathrm{S}$ ' and ' $\mathrm{D}$ ', respectively.

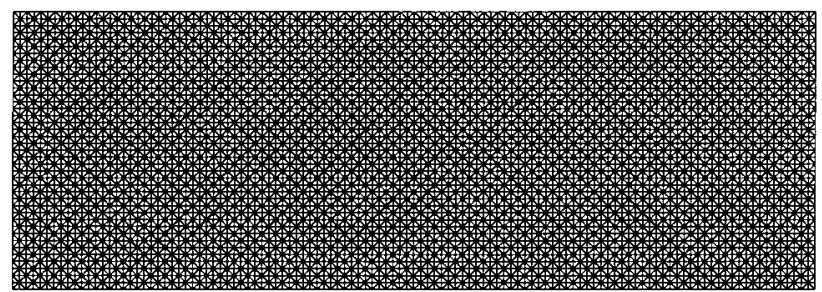

Fig.4 Finite element mesh (4797 nodes and 9280 elements).

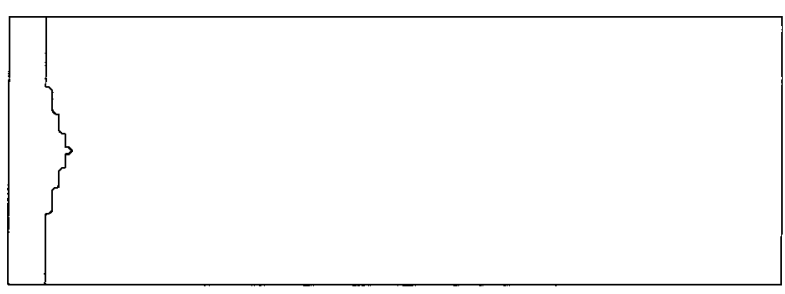

(a)

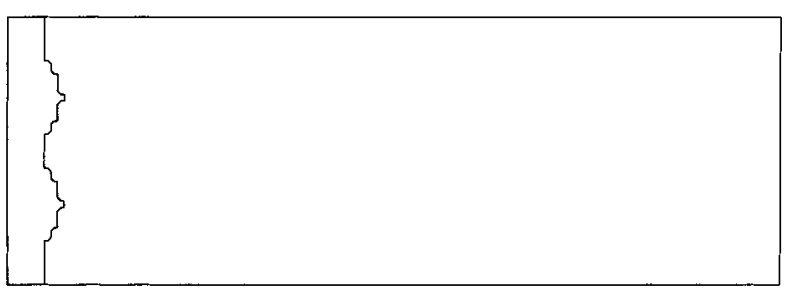

(b)

Fig.5 Initial profile of interface. (a) single protuberance ' $\mathrm{S}$ ', (b) double protuberances ' $\mathrm{D}$ '. 
Firstly, we discuss the growth of viscous fingers in the nonNewtonian fluid from the shape S. In the calculation, we changed $\sigma$ and $U_{\text {ent }}$. Figure 6 shows the results at $U_{\text {ent }}=40 \mathrm{~mm} /$ $\mathrm{s}$ for three values of $\sigma, 40,50$, and $60 \mathrm{mN} / \mathrm{m}$. The interfaces at different time step are superimposed in the figure. The fingertip firstly spreads and after the interface becomes unstable, it splits into two fingers. When $\sigma$ is large, the interface is stable and the occurrence of the splitting is delayed. These results qualitatively agree with experimental observations.

Figure 7 shows the results in the non-Newtonian fluid at three inlet velocities $U_{\text {ent }}$ for $\sigma=75 \mathrm{mN} / \mathrm{m}$. The interfaces at different time step are superimposed in the figure. The splitting is shown in Fig.7(b), (c). When the finger speed increases, the interface becomes unstable thus the finger splits at earlier step.

Next, we investigated the growth from the shape D. Figure 8 shows the results of the finger growth from the doubleprotuberances at $U_{\text {ent }}=50 \mathrm{~mm} / \mathrm{s}$. The fingering patterns in the Newtonian and the non-Newtonian fluids are compared. Strong shielding effect is observed in the case of the nonNewtonian fluid: The shielding is a phenomenon that a finger

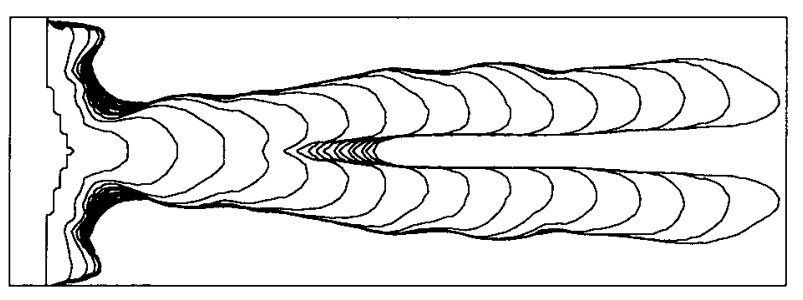

(a) $\sigma=40 \mathrm{mN} / \mathrm{m}$

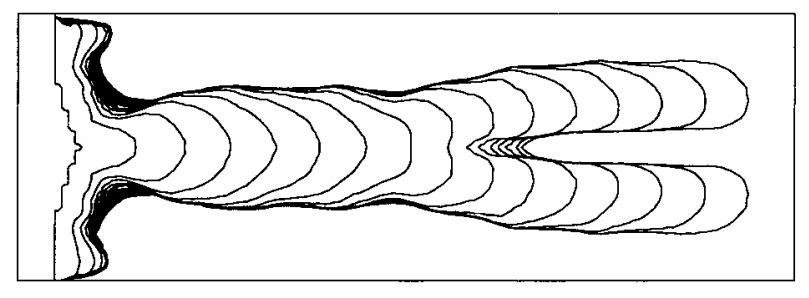

(b) $\sigma=50 \mathrm{mN} / \mathrm{m}$

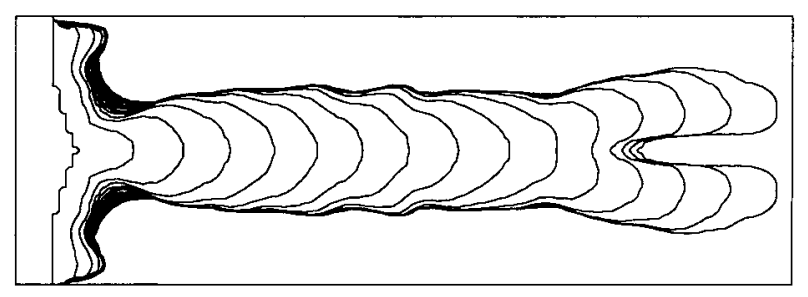

(c) $\sigma=60 \mathrm{mN} / \mathrm{m}$

Fig.6 Viscous fingering patterns in the non-Newtonain fluid at $U_{\text {ent }}=40$ $\mathrm{mm} / \mathrm{s}$. The interfaces at following time steps: (a) $t=0,0.33,0.58$, $0.83,1.07,1.31,1.55,1.80,2.05,2.31,2.58,2.85,3.13,3.41$, $3.69,3.98,4.26 \mathrm{~s}$, (b) $t=0,0.32,0.58,0.82,1.06,1.30,1.54,1.79$, $2.05,2.31,2.58,2.86,3.13,3.41,3.70,3.99 \mathrm{~s}$, (c) $t=0,0.32,0.58$, $0.82,1.06,1.30,1.55,1.80,2.06,2.59,2.32,2.85,3.13,3.41$, $3.70,3.98 \mathrm{~s}$ (from left to right).

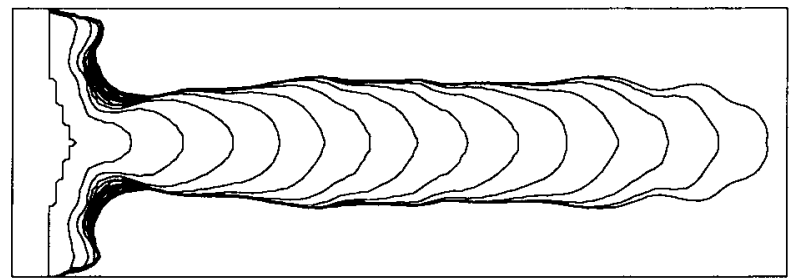

(a) $U_{\text {ent }}=30 \mathrm{~mm} / \mathrm{s}$

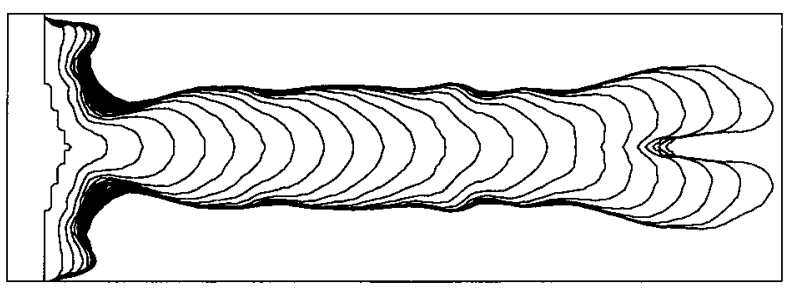

(b) $U_{\text {ent }}=40 \mathrm{~mm} / \mathrm{s}$

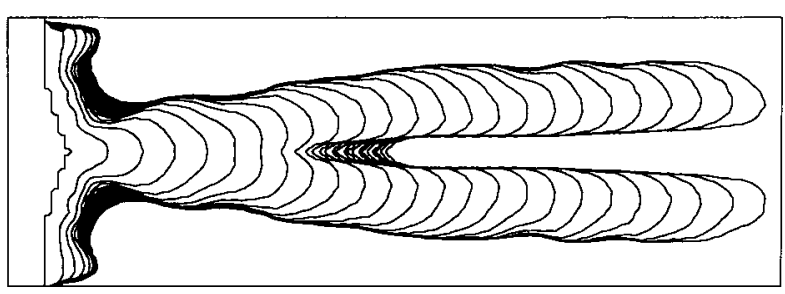

(c) $U_{\text {ent }}=50 \mathrm{~mm} / \mathrm{s}$

Fig.7 Viscous fingering patterns in the non-Newtonain fluid at $\sigma=75$ $\mathrm{mN} / \mathrm{m}$. The interfaces at following time steps: (a) $t=0,0.44,0.78$, $1.10,1.43,1.75,2.09,2.44,2.79,3.15,3.52,3.89,4.26,4.64,5.00$ $\mathrm{s}$, (b) $t=0,0.23,0.40,0.56,0.72,0.87,1.03,1.18,1.34,1.50,1.67$, $1.83,2.00,2.17,2.35,2.53,2.70,2.88,3.06,3.24,3.42,3.61$, $3.79,3.98 \mathrm{~s}$, (c) $t=0,0.19,0.33,0.46,0.59,0.72,0.85,0.97,1.10$ $1.24,1.37,1.51,1.65,1.79,1.93,2.08,2.23,2.37,2.52,2.67$, $2.82,2.98,3.14,3.29,3.44 \mathrm{~s}$ (from left to right).

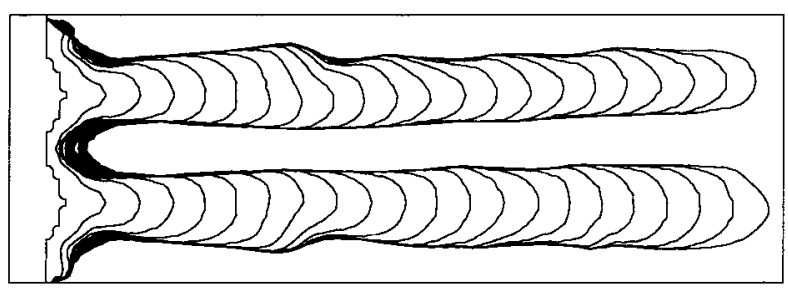

(a) Newtonian

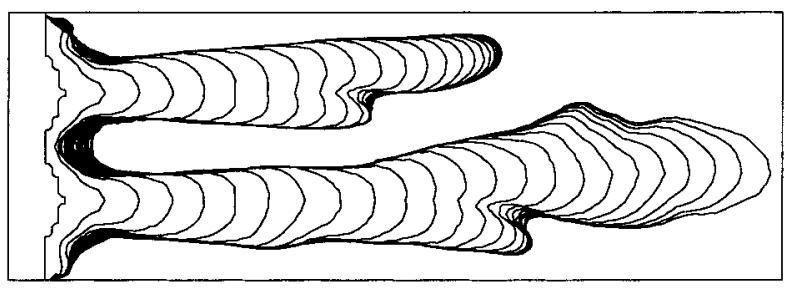

(b) non-Newtonian

Fig.8 Viscous fingering patterns at $U_{\text {ent }}=50 \mathrm{~mm} / \mathrm{s}$ and $\sigma=75 \mathrm{mN} / \mathrm{m}$. The interfaces at following time steps: (a) $t=0,0.22,0.38,0.55,0.72$, $0.90,1.09,1.27,1.44,1.61,1.79,1.96,2.14,2.31,2.49,2.66$, $2.83,3.00,3.18,3.37,3.54,3.71 \mathrm{~s}$, (b) $t=0,0.22,0.38,0.55,0.72$, $0.90,1.09,1.29,1.47,1.65,1.82,1.98,2.14,2.29,2.43,2.56$, $2.68,2.80,2.92,3.02,3.13,3.24,3.34,3.45 \mathrm{~s}$ (from left to right). 
that grows more than other fingers suppresses their growth. Because of the shear-thinning viscosity, small difference in the pressure gradient near two finger fronts causes large difference in viscosity, thus the shear-thinning viscosity increases the shielding effect.

As indicated above, the present simulation shows the typical growth patterns and the prediction of the effect of the shearthinning viscosity on the shielding qualitatively agrees with experimental results. However, the simulation has not predicted some phenomena observed in our experiments ${ }^{12,15)}$ such as the structural change that thin fingers suddenly grows from relatively wide finger-tips, hence we should modify the simulation scheme in future study.

\subsection{Velocity vectors}

We investigated the velocity field in the high viscous fluid region. Although, the velocity is effective information in flow analyses, the velocity measurement in the viscous fingering in a Hele-Shaw cell is not easy.

Figures 9 and 10 show the change in velocity vectors from the initial shapes $\mathrm{S}$ and $\mathrm{D}$, respectively: The high viscous fluid is the non-Newtonian fluid and $\sigma$ is $75 \mathrm{mN} / \mathrm{m}$. The mean inlet velocity $U_{\text {ent }}$ is $50 \mathrm{~mm} / \mathrm{s}$ for each calculation. The vectors consist of $\bar{u}$ and $\bar{v}$ as the $x$ and $y$ components, respectively.

In Fig.9, the velocity is large near the finger-tip and the direction of the velocity vectors indicates that the interface tends to spread. When the finger-tip spreads largely, the interface stability is broken and the tip begins to split. An indication of the splitting is shown in Fig.9(b). After the interface splits, the velocity between the projecting two fingers decreases and the interface in this region stops growing. On the other hand, the velocity near the two fingers is large and the fingers advance. The finger-tips do not spread widely after the splitting because there is not enough space either between two fingers or between each finger and the wall. No splitting follows the first one. Under the present condition, the shielding does not occur obviously.

Similarly to the finger growth from the single-protuberance, the velocity is large near the each finger-tip and the front interface tends to spread. An indication of the splitting is observed at the tip of the upper finger (Fig.10(c)). The shielding phenomenon follows the splitting. One finger restricts the other finger's growth and the velocity near the forward finger is larger.

The velocity near the upper finger-tip is much smaller than that near the lower finger (Fig.10(d)). Consequently, the lower finger advances more than the upper one. The advanced finger spreads and restrains the growth of the upper finger and the upper finger finally stops growing (Fig.10(e) and (f)). The splitting and shielding are also observed for the lower finger.

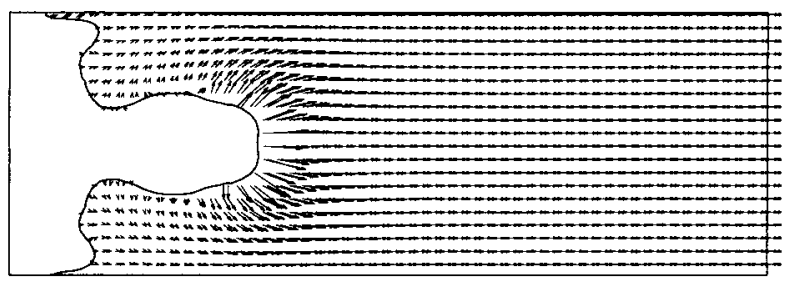

(a)

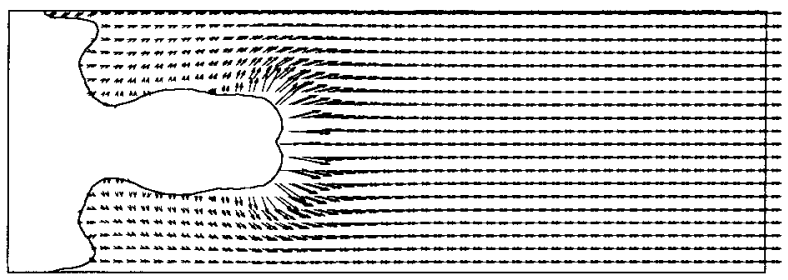

(b)

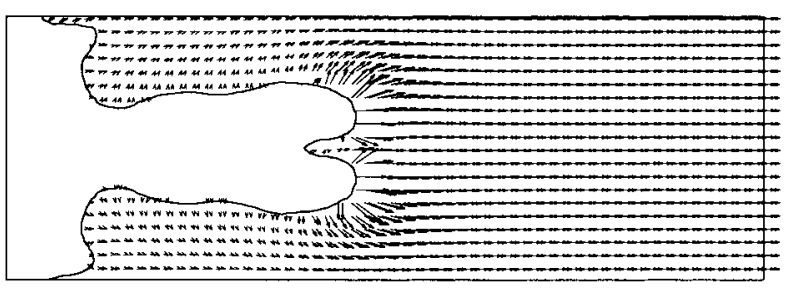

(c)

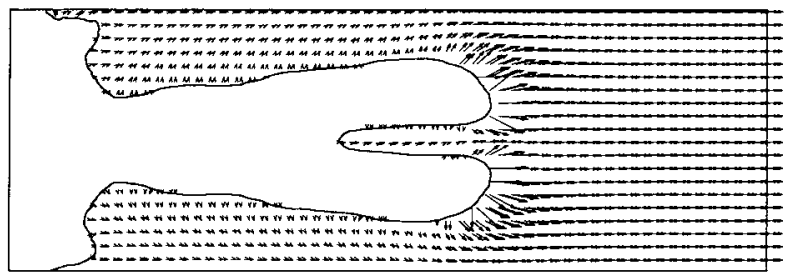

(d)

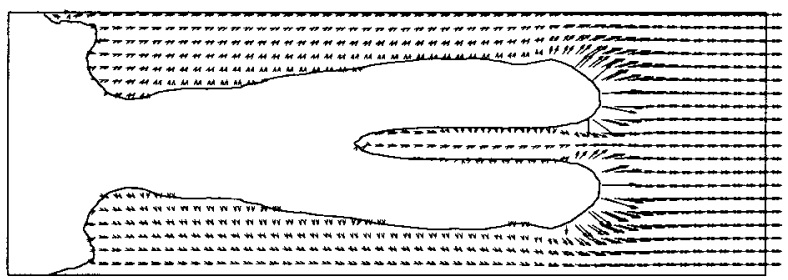

(e)

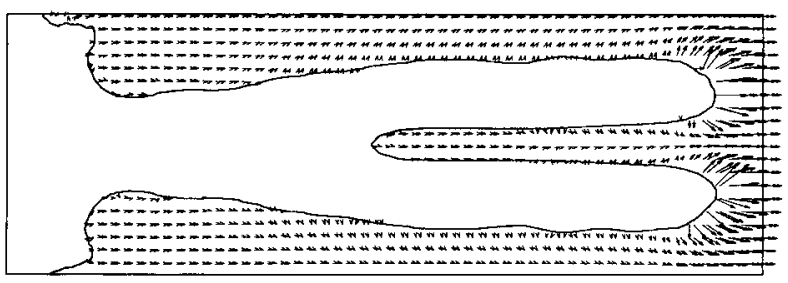

(f)

Fig.9 Velocity vectors for viscous fingering in the non-Newtonian fluid at $t=0.85,0.97,1.37,2.08,2.67,3.29 \mathrm{~s}$ (from top to bottom): $U_{\text {ent }}=50 \mathrm{~mm} / \mathrm{s}, \sigma=75 \mathrm{mN} / \mathrm{m}$. 


\section{CONCLUSION}

In the present study, we simulated the viscous fingering in the rectangular cell using FEM together with the VOF method

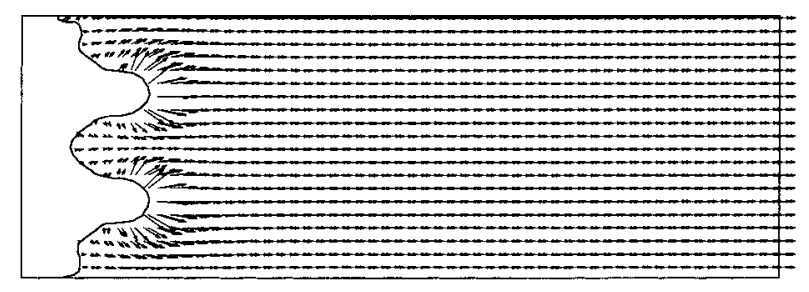

(a)

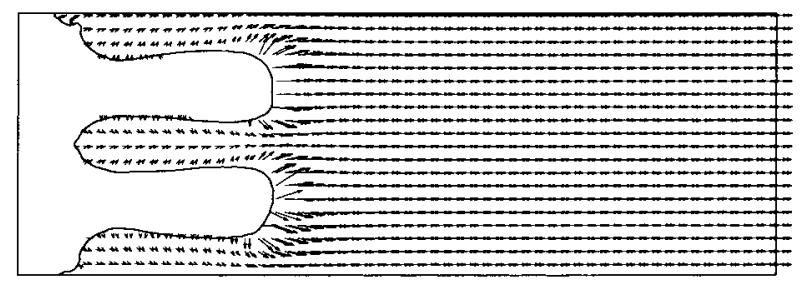

(b)

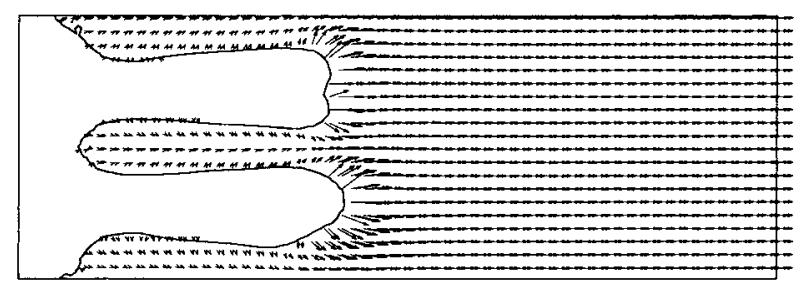

(c)

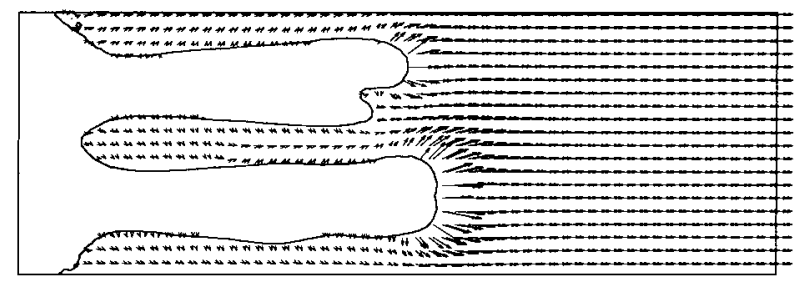

(d)

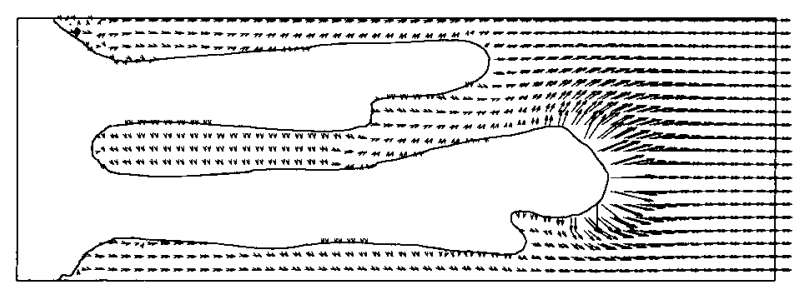

(e)

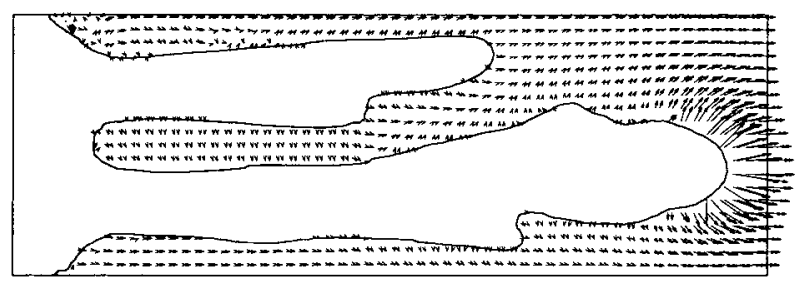

(f)

Fig.10 Velocity vectors for viscous fingering in the non-Newtonian fluid at $t=0.38,1.09,1.47,2.29,2.80,3.34 \mathrm{~s}$ (from top to bottom): $U_{\text {ent }}=50 \mathrm{~mm} / \mathrm{s}, \sigma=75 \mathrm{mN} / \mathrm{m}$. for the movement of the interface and the CSF model for the interfacial force. The computational results showed the availability of the present approach for simulating the viscous fingering in non-Newtonian fluids: The numerical results qualitatively predicted the typical phenomena such as shielding, spreading, and splitting and the effect of shearthinning viscosity. Furthermore, effective information such as the velocity was obtained. In future study, we should introduce more precise expression for the interface and take the effect of elasticity into account to modify the computational model.

\section{REFERENCES}

1) Homsy GM, Ann Rev Fluid Mech, 19, 271 (1987).

2) Feder J, "FRACTALS", (1988), Plenum Press, New York, Chapter 4.

3) Vicsek T, "Fractal Growth Phenomena", (1989), World Scientific Publishing, Singapore.

4) Tanveer S, J Fluid Mech, 409, 273 (2000).

5) Tosaka N, Miyata H, Shimazaki Y, Nomura T, Shimura M, Hatanaka K, “Analysis of Moving Boundary Flows”, (1995), Tokyo University Press, Tokyo, Chapter 4 (in Japanese).

6) Nakayama T, Motogami S, Fluid Dyn Res, 22, 73 (1998).

7) Lu X, Chiang HH, Fong L, Zhao J, Chen SCS, Polym Eng Sci, 39, 62 (1999).

8) Wilson SDR, J Fluid Mech, 220, 413 (1990).

9) Smith DE, Wu XZ, Libchaber AL, Moses E, Witten T, Phys $\operatorname{Rev} A, 45, \mathrm{R} 2165$ (1992).

10) Sader JE, Chan DYC, Hughes BD, Phys Rev E, 49, 420 (1994).

11) Yamamoto $T$, Kamikawa H, Nakamura K, Nihon Reoroji Gakkaishi, 24, 105 (1996) (in Japanese)

12) Kamikawa H, Master Thesis, (1996), Osaka University (in Japanese).

13) Bonn D, Meunier J, Phys Rev Lett, 79, 2662 (1997).

14) Weisser EM, Boger DV, Abst 2nd Pacific Rim Conf on Rheology, 297 (1997).

15) Yamamoto T, Kamikawa H, Nakamura K, Abst 2nd Pacific Rim Confon Rheology, 309 (1997).

16) Kawaguchi M, Shibata A, Shimomoto K, Kato T, Phys Rev E, 58, 785 (1998).

17) Amar MB, Poiré EC, Phys Fluids, 11, 1757 (1999).

18) Lindner A, Bonn D, Meunier J, J Phys Condens Matter, 12, A477 (2000).

19) Lindner A, Bonn D, Meunier J, Phys Fluids, 12, 256 (2000).

20) Yamamoto T, Kamikawa H, Tanaka H, Nakamura K, Mori N, Nihon Reoroji Gakkaishi, 29, 81 (2001).

21) Brackbill JU, Kothe DB, Zemach C, J Comput Phys, 100, 335 (1992).

22) Kothe DB, Mjolsness RC, AIAA J, 30, 2694 (1992). 